\section{Les décisions affectant la} \section{recherche durant} une période de restrictions financières: Les soins infirmiers en valent-ils la peine?

\author{
par Lorna Butler, Ph.D.
}

\section{Abrégé}

Lorsqu'un centre hospitalier déclare dans son énoncé de mission qu'il est assujetti à l'excellence en recherche, il convient de considérer la façon dont les ressources existantes sont affectées en vue de développer les initiatives de recherche et d'appuyer le perfectionnement des chercheures cliniques. Cette étude décrit comment le service des soins infirmiers a relevé le défi présenté par l'énoncé de mission de son hôpital en élaborant un programme de recherche en sciences infirmières. Étant donnée la nature décentralisée du service des soins infirmiers qui chapeaute six départements différents, la composante recherche s'est axée sur les soins en oncologie, un des principaux secteurs de pointe dans le domaine de l'excellence clinique dans cet établissement. L'étude présente les róles spécifiques pour la vice-présidente, les directrices de soins infirmiers, les infirmières-chefs, les infirmières cliniciennes spécialisées et les éducatrices; elle se penchera aussi sur l'embauche d'une spécialiste en recherche clinique afin de conceptualiser le modèle et de mettre en oeuvre le processus de recherche.

\section{Introduction}

En ces temps de réforme des soins de santé et de compressions budgétaires, la création de nouveaux postes au niveau d'un département soulève l'attention et peut avoir pour résultat la remise en question des décisions des administratrices concernées. Pour les infirmières administratrices oeuvrant dans des hôpitaux de soins aigus, la mise au point d'un plan stratégique reflétant la mission de l'hôpital en matière d'excellence dans les soins aux patients laisse supposer l'existence d'un modèle de prise de décisions et de formulation des politiques - un modèle fondé sur une information juste et d'actualité. C'est pour répondre à ce défi que les infirmières administratrices ont reconnu l'importance de l'inclusion de programmes de recherche au sein de l'orientation de la pratique clinique, de la formulation des politiques et de la prise de décisions administratives (Smeltzer et Hinshaw, 1993).

La vice-présidente des soins infirmiers du Victoria General Hospital a créé un poste d'infirmière chercheure en dépit des restrictions financières actuelles. La recherche devait avoir comme rôle principal l'élaboration d'un modèle pour la recherche infirmière dans l'un des principaux secteurs d'excellence clinique de l'hôpital. On a retenu I'oncologie comme étant la spécialité clinique sur laquelle porterait la recherche infirmière et celle qui indiquerait la voie à suivre au sein du service des soins infirmiers. Puis on s'est penché sur le processus à mettre en oeuvre pour étudier le degré de préparation d'un établissement relativement au soutien des activités de recherche infirmière; ce processus s'intéressait aussi au modèle de mise en oeuvre et au soutien administratif nécessaire à l'élaboration et au maintien d'une composante de recherche au sein d'un service de soins infirmiers.

\title{
Information générale
}

Le service des soins infirmiers avait mis sur pied un comité de la recherche et les infirmières cliniciennes spécialisées ont rę̧u une période de dégagement pour la recherche. Le comité de la recherche se composait de représentantes des infirmières de chevet, des infirmières-chefs, des infirmières cliniciennes spécialisées, des infirmières-éducatrices et des directrices des soins infirmiers. Outre ce comité, on a créé un poste honoraire en recherche infirmière partagé entre l'hôpital et une membre du corps professoral d'une école en sciences infirmières universitaire. On voulait surtout faire en sorte que les activités de recherche acquièrent une certaine visibilité au sein du service des soins infirmiers pour faire comprendre aux infirmières qu'il leur incombe de remettre en question les procédures et d'adopter une pratique infirmière fondée sur les résultats. On y soulignait aussi l'importance du rôle joué par les infirmières cliniciennes spécialisées en matière de recherche. Et, dans le but de rendre cet aspect de leur rôle encore plus explicite, on a alloué $20 \%$ de leur horaire à la recherche tout en exprimant le désir de vérifier l'efficacité de ce bloc de temps consacré à la recherche.

Le but recherché, à savoir faire de la recherche une composante importante des soins infirmiers, était louable, mais il s'accompagnait d'un défi de taille: il fallait à la fois mener les recherches et aider les infirmières à utiliser les résultats dans la pratique. Les responsables de l'administration infirmière ont déclaré qu'il fallait affecter les ressources de manière appropriée afin que le travail de recherche puisse se faire. II s'agissait là, du point de vue pratique et des connaissances requises, une tâche colossale pour les membres du comité de recherche. Les infirmières cliniciennes spécialisées n'étaient pas non plus épargnées, du fait de leur charge de travail existante. Sur le plan de l'organisation, I'hôpital devait également évaluer comment ses ressources actuelles lui permettraient de répondre aux besoins de la recherche infirmière. À l'intérieur d'un centre de soins tertiaires où l'on se consacre à l'enseignement ainsi qu'aux essais cliniques médicaux et pharmaceutiques, on pourrait penser qu'il existe un grand bassin de soutien pour la recherche. On a soulevé la question de savoir si les ressources existantes pourraient répondre aux besoins des soins

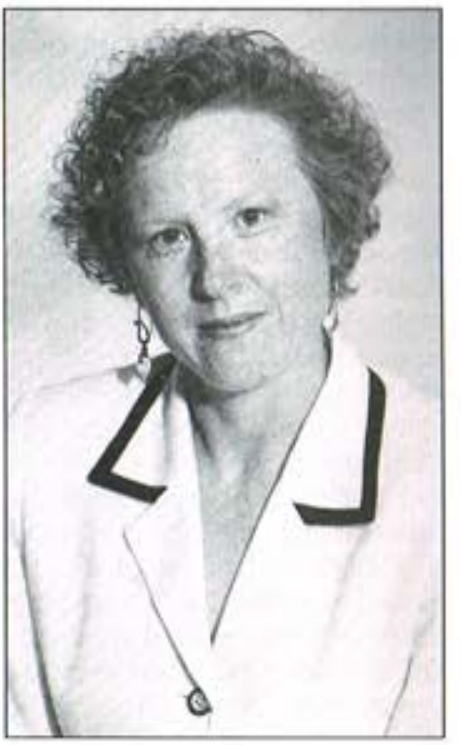

Lorna Butler 
infirmiers et si on pourrait assumer les coûts associés au soutien et au maintien à long terme de la recherche.

La mise en oeuvre de la recherche infirmière dépend d'un certain nombre de facteurs au sein du service des soins infirmiers. Par exemple, dans notre centre hospitalier, $83 \%$ des infirmières sont des infirmières de niveau collégial et parmi elles, 4,6\% étaient inscrites à des programmes de baccalauréat en sciences infirmières. Nous pouvions donc supposer qu'elles étaient nombreuses à n'avoir jamais réalisé de recherches documentaires informatisées ou à en posséder les rudiments. Dans notre bibliothèque, une demande de recherche documentaire s'accompagne de frais. Qui allait payer ces frais, l'unité de soins infirmiers ou la bibliothèque? Combien de demandes serait-on en droit d'attendre de la bibliothèque? II importait aussi de savoir qui enseignerait aux infirmières le savoir-faire en matière de recherche documentaire et la façon dont on allouerait ces heures dans le cas du personnel affecté aux soins aux patients dans les unités. Ceci entraînait une responsabilité supplémentaire pour l'infirmière-chef qui devait rajouter des heures à son budget et pour les éducatrices qui, elles, devraient satisfaire à des demandes accrues de temps et donc d'argent.

Dans sa vision, la vice-présidente des services de soins aux patients réservait une place de choix à la recherche parmi les composantes de son service. Bien qu'à l'origine son plan n'ait pas fait l'objet d'une élaboration poussée, elle s'était efforcée d'adopter une voie raisonnable et viable. La phase initiale consistait à évaluer l'hôpital afin de dégager les obstacles humains et structurels et de tirer profit des atouts existants en vue de déterminer les rôles en recherche infirmière. Dans l'ensemble, on peut dire qu'il y avait d'excellentes ressources, mais que de grands besoins se faisaient sentir en éducation. La plupart des sources de soutien n'avaient pas l'habitude de faire face aux demandes d'infirmières et on se préoccupait de savoir d'où viendraient les ressources nécessaires à la prestation des services. Par exemple, la bibliothèque serait-elle en mesure de répondre à la charge de travail si plusieurs infirmières commençaient à utiliser ses services? Les infirmières éducatrices pourraient-elles affecter une partie de leur temps et de leurs ressources à cette nouvelle fonction? L'accréditation de l'hôpital en 1994 avait révélé qu'en dépit de son nom, la bibliothèque n'était pas une bibliothèque des sciences de la santé mais bien une bibliothèque médicale, ce qui limitait les services offerts aux infirmières. Ainsi, l'accès aux banques de données en sciences infirmières devait se faire à l'université voisine, par le biais d'une recherche documentaire externe. Comme les infirmières de chevet étaient dans l'impossibilité de quitter l'établissement dans le cadre de leur recherche, il fallait tenir compte des coûts additionnels que chaque recherche documentaire externe engendrait pour la bibliothèque et l'unité de soins infirmiers.

Le département des services de documentation a offert un excellent soutien en matière de matériel informatique et des logiciels faisant déjà l'objet d'une utilisation à l'hôpital. Par contre, aucun service n'était disponible pour ce qui est du domaine statistique; le département était dans l'incapacité d'aider le projet à résoudre les problèmes associés aux logiciels achetés dans le but d'effectuer les analyses statistiques. Les soins infirmiers se devaient de relever un défi de taille: établir des liens entre l'hôpital et l'université voisine afin que les infirmières puissent mettre à profit les services et le soutien de recherche dispensés par l'université et le corps professoral. On a signalé qu'il fallait favoriser des partenariats avec les infirmières-chercheures lorsqu'il était impossible de transférer des fonds.

Une autre tâche était d'évaluer la réceptivité et la volonté du service des soins infirmiers de prendre en considération un plan de recherche infirmière. On ne savait pas vraiment dans quelle mesure les infirmières de l'établissement valorisaient la recherche et si elles connaissaient bien l'expression "pratique fondée sur les résultats".

\section{Intégration de la recherche et de la pratique infirmières}

Les membres du comité de recherche infirmière et des représentantes du service des soins infirmiers ont participé à une journée de réflexion afin de décider de l'opérationnalisation de la recherche au sein du service. Le but de la journée de réflexion était d'élaborer les objectifs stratégiques et les indicateurs de succès qui serviraient à déterminer un cadre pour la recherche lequel appuierait le plan stratégique du service. C'est ainsi que les membres du comité ont reçu, à des fins de préparation de la journée, les questions suivantes portant sur les composantes organisationnelles de la recherche (Eagle, Fortnum, Price et Scruton, 1990):

1) Quel cadre de recherche conviendrait au service des soins infirmiers? 2) D'après le service des soins infirmiers, quelle doit être la fonction du comité de recherche?

3) Quelles fonctions seraient associées au rôle de chercheure au sein du service des soins infirmiers?

4) Quels arrangements doit-on prévoir afin de soutenir la recherche par l'intermédiaire de postes conjoints avec l'université?

5) Que doit-on réaliser afin de mettre en place l'infrastructure administrative qui appuiera la recherche?

Cadre de recherche. On a présenté aux participantes de la journée de réflexion quatre cadres pour la recherche afin qu'elles puissent sélectionner ceux qui devraient recevoir la priorité. II s'agissait des quatre cadres suivants: cadre clinique, cadre administratif, évaluation et assurance de la qualité de programmes ainsi qu'un rôle générique. On a demandé aux participantes si tous les cadres de recherche infirmière devaient recevoir la même attention, si les attitudes et les croyances des infirmières vis-à-vis un cadre particulier pouvaient varier en vertu de la valeur accordée actuellement à la recherche infirmière et s'il y avait des ressources infirmières disponibles pour la recherche en vue d'appuyer le cadre qu'elles voulaient sélectionner. On a également demandé aux participantes de faire ressortir les lacunes et les changements qu'il faudrait mettre en place avant d'atteindre les objectifs relatifs au cadre de recherche retenu.

Les participantes se sont alors divisées en petits groupes pour discuter des questions tout en veillant à obtenir une répartition équilibrée des rôles infirmiers (pratique clinique, administration et recherche). On s'est servi d'un système de matrices pour évaluer les quatre cadres de recherche proposés. Les résultats indiquaient que le cadre mentionné le plus fréquemment était celui de la recherche en pratique clinique. L'échelle de pondération de l'importance relative accordée à chaque cadre faisait, elle aussi, ressortir la pratique clinique. On accordait donc la priorité à la pratique clinique, même s'il ne s'agissait pas là d'un choix unanime. Soixante pour cent des membres ont choisi la pratique clinique comme cadre préféré pour la recherche. Pour les résultats à atteindre, les indicateurs de réussite de la recherche en pratique clinique incluaient les thèmes suivants

- le rehaussement de la réflexion et du questionnement

- des questions de pratique clinique soulevées par les infirmières de chevet

- la collaboration entre chercheures et infirmières cliniciennes spécialisées pour une participation active à la recherche

- des résultats pertinents présentant des répercussions pour la pratique

- le partage des résultats - le soutien à la publication

- la reconnaissance de la participation de toutes les collaboratrices

- la démysthification du processus de recherche

Attitudes des infirmières envers le cadre de recherche. Afin d'examiner la question des attitudes des infirmières relatives à l'acceptation d'un cadre de recherche clinique, on a demandé aux groupes de réfléchir sur les divers rôles que l'on retrouve au sein du service des soins infirmiers, sur les différents aspects du processus de recherche et sur ce que les membres du service devraient faire pour atteindre le but de la recherche. Elles ont fait ressortir les principales responsabilités qu'elles devraient assumer. II est intéressant de constater que le rôle du personnel administratif a été clairement défini tandis que ce n'était pas vraiment le cas pour celui des infirmières oeuvrant directement dans les différents champs de la pratique clinique. Les opinions différaient quand il s'agissait de déterminer qui mènerait la recherche et qui participerait à la recherche ou en assurerait la coordination. Puis, afin de clarifier les attentes relatives au soutien 
disponible au sein du service des soins infirmiers, on a défini les rôles et les responsabilités en matière de conduite et d'utilisation de la recherche. Les membres sont parvenues au consensus dans toutes les catégories (voir le tableau 1).

On a examiné les forces internes du service de soins infirmiers qui permettent d'appuyer la recherche. Lorsque les participantes ont examiné les ressources infirmières existantes pour décider si elles pourraient fournir l'expertise nécessaire à la mise en oeuvre du cadre de recherche clinique, les avis étaient partagés. La dernière question portait sur les changements qu'il faudrait mettre en place pour pouvoir appuyer la recherche au sein du service et parmi les autres intervenants de la santé représentés à l'hôpital. On a relevé les domaines suivants comme nécessitant les changements les plus importants:

- embaucher une infirmière chercheure

- établir des liens entre le service des soins infirmiers et l'école en sciences infirmières universitaire pour les activités de recherche

- engager des ressources financières comme de petites subventions pour la mise en oeuvre de la recherche

- déterminer la valeur accordée à la recherche et l'attitude des infirmières travaillant au sein du service envers la conduite de la recherche et I'utilisation des résultats

- reconnaître les initiatives d'infirmières oeuvrant à des travaux d'érudition telles que des publications

- inviter les étudiantes en sciences infirmières des deuxième et troisième cycles à réaliser leur recherche au sein de l'hôpital et les soutenir dans leurs efforts.

Les infirmières gestionnaires représentant les différentes sciences de la santé se réunissaient régulièrement dans le cadre du Conseil de la vice-présidente. Toutes avaient la volonté de travailler ensemble, mais aucune initiative n'avait encore vu le jour. En outre, on a signalé le fait qu'il n'y avait aucune structure de soutien administratif visant à faciliter ou à coordonner la recherche interdisciplinaire au sein du Conseil.

Il fallait également apporter des changements de taille à d'autres éléments de soutien présents dans l'hôpital même: services de bibliothèque, services de documentation / d'informatique pour le soutien en matière de programmation et de données statistiques, accès aux services d'arts graphiques, systèmes de couplage des données et soutien audio-visuel pour la dissémination des résultats par le biais de présentations d'affiches et de diaporamas. De même, les ressources disponibles dans les universités voisines et dans le Centre de recherche clinique de I'hôpital nécessitaient des changements afin de favoriser la recherche clinique (le Centre appuyait surtout les projets de recherche sous contrat). Nul doute que ces changements étaient nécessaires pour les ressources existantes, mais le service des soins infirmiers devait aussi commencer à faire preuve d'innovation afin de pouvoir accéder à la structure de soutien en termes de financement et de responsabilité financière.

\section{Discussion}

Pour ce qui est des hôpitaux canadiens, les programmes de recherche clinique en sciences infirmières réalisés en milieu hospitalier sont très récents et n'en sont encore qu'aux phases de démarrage (Fitch, 1992; Thurston, Tenove, Church et Bach-Paterson, 1989). La mise en oeuvre d'un tel programme et l'assurance de sa viabilité dépendent presque entièrement de l'engagement de l'administration à négocier en faveur de la recherche

infirmière et de la volonté de fonder la prise de décisions sur des assises scientifiques (Marchette, 1985). Les responsables des soins infirmiers ne doivent pas non plus perdre de vue la signification, dans leur établissement, de la pratique fondée sur la recherche. Elles doivent se demander quelles sont les composantes essentielles pour l'élaboration d'un plan stratégique qui permettra de concrétiser la vision qu'elles ont de la recherche et quel impact celle-ci aura sur la pratique clinique des infirmières (Fitch, 1992).

Les étapes franchies par les infirmières de cet établissement révélaient la présence d'un important soutien administratif en vue de mettre au point une composante de recherche au sein du service des soins infirmiers. La première étape en vue d'y parvenir a été l'embauche d'une infirmière chercheure à temps plein dont la priorité initiale était de structurer et d'élaborer l'environnement hospitalier nécessaire à la reconnaissance et à l'acceptation de la recherche infirmière. Ceci a

Tableau 1 : Attentes du service des soins infirmiers en vue d'appuyer la recherche

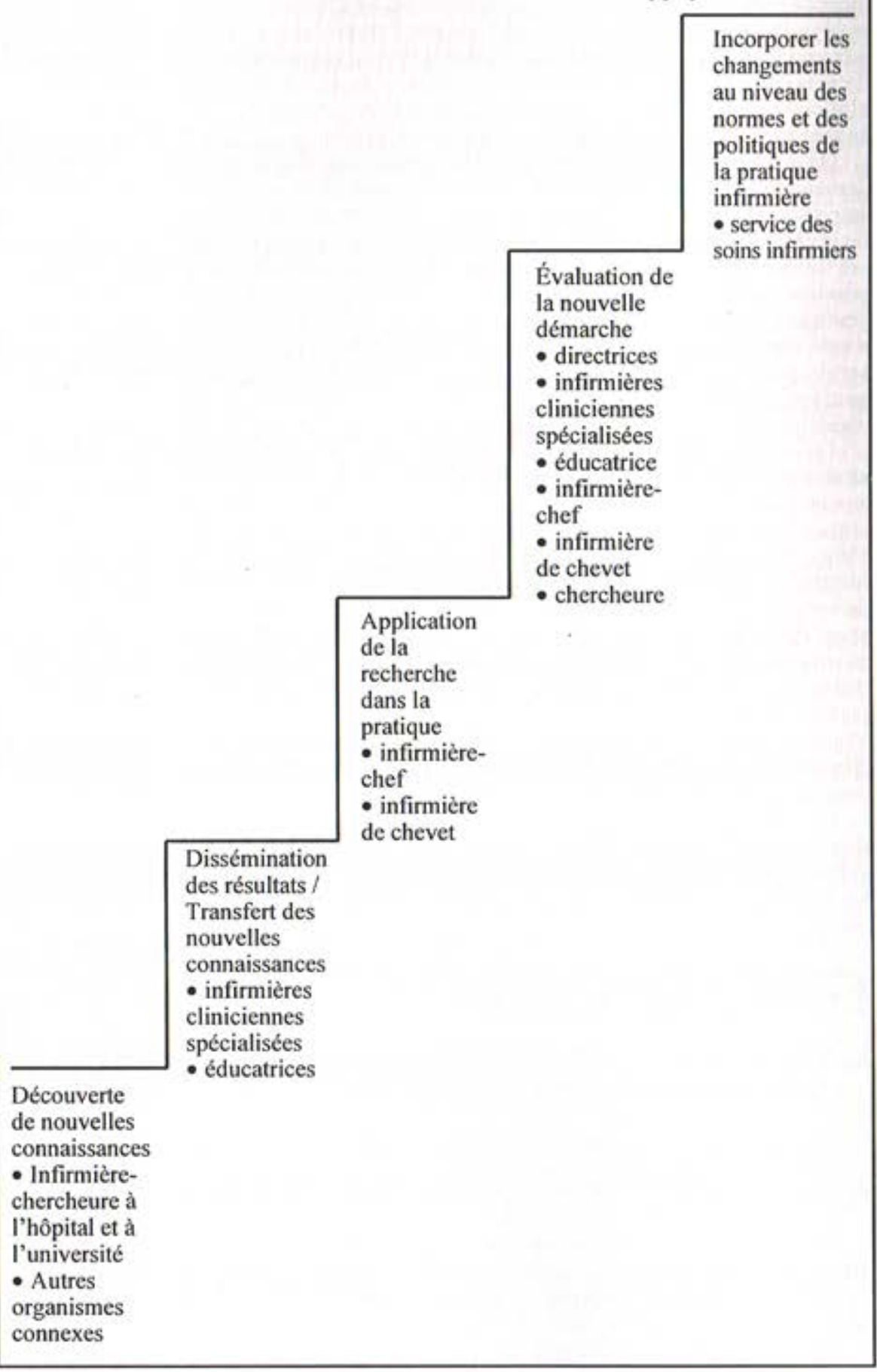


permis de renforcer les rôles et responsabilités du comité de recherche infirmière. Les soins infirmiers avaient bien une voix au comité d'évaluation de la recherche de l'hôpital, mais la représentation était laissée au bon vouloir des intéressées. Le comité de la recherche infirmière s'est empressé de déléguer une représentante auprès du comité d'évaluation, ce qui lui a permis d'opérationnaliser l'orientation stratégique de cette facette de l'objectif des soins infirmiers en matière de recherche (Vessey et Campos, 1992).

Le rôle des infirmières cliniciennes spécialisées comporte diverses exigences et charges de travail difficiles en vue de développer la pratique des soins infirmiers. II y a lieu de féliciter l'administration d'avoir pris l'initiative de réserver à la recherche une partie de l'horaire des infirmières cliniciennes spécialisées. Cependant, on peut dire qu'il s'agissait d'un plan ambitieux: sans système d'accès aux ressources et sans orientation clairement définie pour la recherche infirmière en pratique clinique, on plaçait d'énormes attentes sur le dos de ces infirmières dont on ne savait pas vraiment si elles pourraient ou non les mener à bien. II fallait discuter en permanence de la différence entre les responsabilités associćes à l'utilisation des résultats et celles associées à la conduite des projets de recherche. On a décidé que la recherche demeurerait une composante prioritaire du rôle des infirmières cliniciennes spécialisées; on a toutefois différé la mesure d'un engagement de temps en terme de résultat de recherche.

On a retenu le domaine des soins infirmiers en oncologie comme domaine de pratique clinique qui verrait l'élaboration et la mise en ocuvre des initiatives de recherche infirmière. C'est conjointement avec les infirmières cliniciennes spécialisées que les infirmières en oncologie ont commencé à soulever les questions de pratique clinique qui se posaient dans leur travail quotidien. Pour passer des questions de pratique à un forum de sujets potentiels de recherche, la vice-présidente a créé une subvention de recherche dont les infirmières pouvaient se servir pour commencer leurs études. Les infirmières avaient la motivation requise pour participer, mais il leur manquait encore direction et formation au niveau du processus de recherche. Il importait d'obtenir une masse importante d'infirmières qui seraient non seulement excitées à l'idée de participer à la recherche, mais qui auraient aussi les connaissances nécessaires et seraient intéressées à utiliser les résultats de la recherche dans leur pratique (Bostrom et Wise, 1994). Pour obtenir un tel groupe d'infirmières, il fallait que les ressources disponibles au niveau des soins infirmiers incluent la recherche infirmière au sein de leurs responsabilités en matière de rôle et qu'elles apportent leur aide au niveau des possibilités d'éducation permanente. Les infirmières éducatrices, gestionnaires et administratrices ainsi que les infirmières cliniciennes spécialisées jouaient un rôle vital au niveau de la facilitation de tels programmes; toutefois, ce sont les infirmières de chevet qui forment la véritable clientèle des projets de recherche et on avait à peine commencé à étudier leurs besoins.

C'est pour déterminer les besoins d'apprentissage des infirmièresdu service de soins infirmiers et pour mettre en oeuvre un plan de recherche pertinent que l'on a mené une étude au sein de l'hôpital afin d'examiner les connaissances des infirmières et la valeur qu'elles accordaient à la pratique des soins infirmiers fondée sur la recherche. Il est apparu que

\section{Bibliographie}

Bostrom, J., Wise, L. (1994). Closing the gap between research and practice. Journal of Nursing Research, 24(5), 22-27.

Butler, L. (1995). Valuing research in clinical practice: A basis for developing a strategic plan for nursing research. Journal of Nursing Research, 27(4), 33-49.

Eagle, J., Fortnum, D., Price, P., Scruton. (1990). Developing a rationale and recruitment plan for a nurse researcher. Canadian Journal of Nursing Administration, 3(2), 5-10.

Fitch, M.1. (1992). Fostering research growth in a nursing department. Canadian Journal of Nursing Administration, 9(1), 11-27.

Jairath, N. (1990). Nursing research organizational models. Canadian Journal of Nursing Administration, 3(2), 11-16. les infirmières croyaient dans la pratique fondée sur les résultats empiriques mais qu'elles possédaient bien peu d'expérience au niveau de la conception de leurs propres idées de recherche. Dans la plupart des cas, l'expérience des infirmières se limitait à l'adoption d'un rôle de soutien envers les chercheurs menant des recherches axées sur les essais cliniques dans les unités de soins. Les résultats de cette étude sont présentés dans un autre document (Butler, 1995).

\section{Conclusion}

La réforme des soins de santé exige que l'on fasse appel à la créativité et à l'innovation afin de dispenser les soins d'une manière plus efficace et plus rentable. On remet plus que jamais en question la prestation des soins de santé et on exige des sciences de la santé qu'elles relèvent le défi ainsi lancé. Le processus de recherche permet aux infirmières de soulever des questions, de vérifier des idées, d'explorer des solutions de rechange et de plaider la cause du changement. Pour y parvenir, les infirmières doivent valoriser la pensée critique, encourager les idées nouvelles et se risquer à explorer des territoires inconnus tout en gardant l'esprit ouvert. La recherche n'est plus un ornement. Elle représente un lien essentiel et intégral de la définition du rôle en soins infirmiers au sein de la politique de la santé et du processus de prise de décisions en matière de prestation des soins que l'on connaîtra à l'avenir.

Le centre hospitalier a examiné les ressources et les a réaffectées afin qu'elles fassent l'objet d'une utilisation adéquate par toutes les disciplines de la santé. Les coûts sont restés moindres étant donné que les changements se sont surtout produits au niveau des discussions et des croyances philosophiques portant sur la recherche au sein de l'organisme. Depuis le lancement de ce plan de recherche, l'hôpital a fusionné avec trois autres centres de soins. La recherche infirmière a survécu à ce regroupement grâce au profond engagement de la vice-présidente des soins infirmiers. Une partie de la force qui sous-tend cette survie vient du travail et de l'enthousiasme des infirmières de chevet oeuvrant en oncologie qui mènent activement des travaux de recherche sur les soins infirmiers en oncologie.

\section{Remerciements}

L'auteure tient à remercier la Direction des soins infirmiers pour l'orientation stratégique et la vision qu'elle lui a procurées tout au long de la phase d'élaboration du programme de recherche. Elle adresse des remerciements particuliers à $M$. Rowe, $B N$, MEd, vice-présidente de la Direction des soins infirmiers, Victoria General Hospital, et Florence Bell, B.Sc.Inf., MA, coordonnatrice du processus de planification stratégique, Les membres du comité de la recherche infirmière et les participantes à la journée de réflexion sur la recherche ont contribué à la réalisation des objectifs élaborés pour la recherche infirmière. L'auteure remercie également Christine Powers, B.Sc.Inf., MHSA, vice-présidente du Service des soins infirmiers du Queen Elizabeth II Health Sciences Centre pour sa connaissance approfondie en la matière et pour l'orientation qu'elle a donnée pour la recherche infirmière clinique de demain. Quant à Colleen Clattenburg, l'auteure hui témoigne sa reconnaissance pour l'avoir aidée à préparer le manuscrit.
Marchette, L. (1985). Developing a productive nursing research program in a clinical institution. The Journal of Nursing Administration, 15(3), 25-30.

Smeltzer, C.H., Hinshaw, A.S. (1993). Integrating research in a strategic plan. Nursing Management, 24(2), 42-44.

Thurston, N. Tenove, S., Church, J., Bach-Peterson, K. (1989). Nursing research in Canadian hospitals. Canadian Journal of Nursing Administration, 2(1), 8-10.

Vessey, J.A., Campos, R.G. (1992). The role of nursing research committees. Nursing Research, 41(4), 247-249. 